\title{
Study of the Central Heavy-Ion Collision with Emulsion at 4.5 A GeV/c
}

\author{
Alblomy Abd El-Daiem ${ }^{1}$, Mohamed Abdo Khalifa ${ }^{2}$, Atef Abdel-hafiez \\ ${ }^{1}$ Sohag University, Sohag, Egypt \\ ${ }^{2}$ Tanta University, Tanta, Egypt \\ ${ }^{3}$ Nuclear Research Center, Cairo, Egypt \\ E-mail:Abdel_hafiez@yahoo.com \\ Received February 11, 2011; revised March 14, 2011; accepted April 12, 2011
}

\begin{abstract}
We have investigated the main experimental characteristics (multiplicity correlation, angular distributions and the analysis of emission of the shower particles produced at target fragments in central collisions of ${ }^{28} \mathrm{Si}$ $+\mathrm{AgBr}$ at $4.5 \mathrm{AGeV} / \mathrm{c}$ has shown a formation of hadronic and baryonic clusters. Events of central collisions are defined, here, as those having no projectile charged fragments, even a singly charged one, emitted within $3^{0}$ of the beam direction.
\end{abstract}

Keywords: Multiplicity Correlation, Multiplicity Distributions, Hadronic Clusters, Baryonic Clusters

\section{Introduction}

The interaction is central when the impact parameter is less then the absolute value of the difference between the interacting nuclei radii, i.e., $b<\left|R_{\mathrm{P}}-R_{\mathrm{T}}\right|$, where $R_{\mathrm{P}}$ and $R_{\mathrm{T}}$ are the projectile and target nucleus radii, respectively. Such events are characterized by large multiplicities of the generated particles and the emitted target fragments. The emission of particles is symmetric with respect to the direction of the incident beam. In central collision all projectile nucleons are participants and not projectile spectators. The study of the correlation in complete destruction of $\mathrm{AgBr}$ emulsion nuclei is very interesting. This interest forms the fact that most of these interactions are due to central collisions. Central collisions provide a unique opportunity to investigate the consequences of nuclear compression, such as hydro dynamical effect [1-3]. Also, one can study the mechanism of multiparticle production [4-9]. In addition there is a good possibility to obtain valuable information on the excitation and subsequent decay of residual target nucleus. Some theoretical models [10] imply an increase of nuclear matter density in central nucleus-nucleus collisions at high energies. This increase enables the appearance of heterophase state of nuclear matter including a small contribution of quarkgluon phase. According to quantum mechanical ideas, the nucleons participating in multiparticle production will acquire greater size due to their excitation. The previously enumerated conditions should produce an intensive mixing of color degrees of freedom of nucleons constituting the excited nuclear matter. The discoloring process need certain time [11] during which an intermediate system may be formed this system will collect nucleons in its way inside the nucleus which lead to observation of baryonic "jets" or clusters in the process of target nucleus destruction. In the present study is restricted to event of $N_{\mathrm{h}} \geq 28\left(N_{\mathrm{h}}=n_{\mathrm{g}}+n_{\mathrm{b}}\right)$ i.e. to central collisions of ${ }^{28} \mathrm{Si}$ with $\mathrm{AgBr}$, where $N_{\mathrm{h}}$ is the number of heavy target fragments, $n_{\mathrm{g}}$ is the number of grey particles $n_{\mathrm{b}}$ is the number of black particles and we provide a systematic, statistically significant identification of hadronic and baryonic clusters formed in central ${ }^{28} \mathrm{Si}+\mathrm{AgBr}$ collisions at $4.5 \mathrm{~A}$ $\mathrm{GeV} / \mathrm{c}$.

\section{Experiment Details}

Stacks of NIKFI-Br2 nuclear emulsions were exposed to $4.5 \mathrm{~A} \mathrm{GeV} / \mathrm{c}^{28} \mathrm{Si}$ beam at the Dubna synchrophasotron. The stacks have dimension of $20 \mathrm{~cm} \times 10 \mathrm{~cm} \times 0.6 \mathrm{~mm}$. The intensity of irradiation was $10^{4}$ particle $/ \mathrm{cm}^{2}$, and the beam diameter was about $1 \mathrm{~cm}$. Along the track double scanning was carried out, fast in the forward direction and slow in the backward direction. The scanned beam tracks were further examined by measuring the delta electron density on each of them to exclude the track having charge less than beam particle charge. In the 
measured events, the secondary particles are classified as follows: (i) black particle tracks (b) having a range $L<3$ $\mathrm{mm}$ in emulsion which corresponds to a proton kinetic energy of $<26 \mathrm{MeV}$, (ii) grey particle tracks (g) having relative ionization $I\left(=I / I_{0}\right)>1.4$ an $L>3 \mathrm{~mm}$ which corresponds to a proton kinetic energy of $26-400 \mathrm{MeV}$, where $I$ is the particle track ionization and $I_{0}$ is the ionization of a shower track in the narrow forward cone of an opening angle of $\theta \leq 3^{0}$ the $\mathrm{b}$ and $\mathrm{g}$ particle tracks are called heavy ionizing particle tracks (h), and (iii) shower particles (s) having $I<1.4$ tracks of such type with an emission angle of $\theta \leq 3^{0}$ were further subjected to rigorous multiple scattering measurement for momentum determination and consequently, for separating the produced pions and singly charged projectile fragments (protons, deutrons, tritons). (iv) The multi-charged $Z \geq 2$ projectile fragments are subdivided into $Z=2,3, \cdots$, $Z_{\text {beam, }}$ according to the measured delta electron. Thus all particles were adequately divided into projectile fragments of $Z=1$ to $Z_{\text {beam }}$, target fragments (i.e., h particles) and the generated $\mathrm{s}$ particles. The total chsarge of the projectile fragments $Z^{*}=\sum n_{\mathrm{i}} Z_{\mathrm{i}}$ was calculated in each event, where $n_{\mathrm{i}}$ is the number of fragments of charge $Z_{\mathrm{i}}$ in an event. For each track, the following were obtained from the measurements: (a) the polar angle $\theta$, i.e., the space angle between the direction of the beam and that of the given track, and (b) the azimuthal angle $\psi$, the beam and an arbitrarily selected reference direction perpendicular to the beam on this plane (in an anticlockwise direction).

\section{Multiplicity Correlation in Complete Destruction of ${ }^{28} \mathrm{Si}$ Ions}

In the present work, we studied the correlation of complete destruction $\left(N_{\mathrm{h}} \geq 28\right)$ of $\mathrm{AgBr}$ emulsion nuclei induced by $4.5 \mathrm{~A} \mathrm{GeV/c}{ }^{28} \mathrm{Si}$ nuclei. The probability of complete destruction of $\mathrm{AgBr}$ nuclei different projectile at various energies is shown in [12]. The correlation in complete destruction dependencies between the charge particle multiplicities allow us to discuss the mechanism of nucleus-nucleus interactions. The dependencies $<n_{\mathrm{g}}>$ $=f\left(n_{\mathrm{s}}\right)$ and $<n_{\mathrm{s}}>=f\left(n_{\mathrm{g}}\right)$ for the event with $N_{\mathrm{h}} \geq 28$ accompanied by total target disintegration are presented in

Figure 1. and Table 1. in this case there is no strong dependence of $\left\langle n_{\mathrm{g}}>\right.$ on the value of $n_{\mathrm{s}}$ or of $\left\langle n_{\mathrm{s}}>\right.$ on the value of $n_{\mathrm{g}}$. This can be seen from the value of $\chi^{2}$ for each type of particle. This means that the degree of disintegration of the target does not depend strongly on the number of shower particles. One can observe the correlation between the fast and the slow stages of the inelastic interactions of tow nuclei by studying the dependencies $<n_{\mathrm{b}}>=f\left(n_{\mathrm{s}}\right),<n_{\mathrm{b}}>=f\left(n_{\mathrm{g}}\right)$ and $\left(n_{\mathrm{s}}\right),\left(n_{\mathrm{g}}\right)$ on the $\left(n_{\mathrm{b}}\right)$. From Figure 1. one can see that the correlations have a different character. This can be seen from the fast that the $<n_{\mathrm{b}}>$ dependencies on $n_{\mathrm{s}}$ and $n_{\mathrm{g}}$ have negative slopes. Also, from the correlation dependencies of $\left\langle n_{\mathrm{s}}\right\rangle,\left\langle n_{\mathrm{g}}\right\rangle$ and $<n_{\mathrm{h}}>$ on $n_{\mathrm{b}}$, one can see that the slope of $\left.<n_{\mathrm{h}}\right\rangle$ is positive while the slopes of others are negative. From the above results, one can see that $\left\langle n_{\mathrm{b}}\right\rangle$ has a negative correlation with $n_{\mathrm{s}}$ and $n_{\mathrm{g}}$ and the correlation of $\left\langle n_{\mathrm{s}}\right\rangle$ and $<n_{\mathrm{g}}>$ with the $n_{\mathrm{b}}$ is negative. This may be due to increase the number of interacting projectile nucleons as the impact parameter decrease.

\section{Angular Distributions of Target Fragments}

Figure 2. presents the angular distribution of greyparticles emitted in central collisions of ${ }^{28} \mathrm{Si}$ ions with emulsion at $4.5 \mathrm{GeV} /$ nucleon. This distribution compared with the distribution of grey-particles from all ${ }^{28} \mathrm{Si}$ interactions is presented in same figure. It is seen, as has been noted in many papers [13-15], that the angular distribution of grey-particle tracks is very weakly dependent on the variation of projectile mass, the primary energy and the target mass. The number of participant nucleons increases as the volume of the cylinder cut by the projectile in the target nucleus increases. As the mass of the projectile increases, the cross sectional area of this cylinder increases and consequently the number of participants also increases. The size of the target nucleus is limited and consequently the number of evaporated particles decreases when $n_{\mathrm{g}}$ increases. Figure 3. shows the

Table 1. Results of approximate fit of the experimental data for the multiplicity correlation from complete destruction in ${ }^{28} \mathrm{Si}$ ions interactions with emulsion using the dependence $<\boldsymbol{n}_{\mathbf{i}}>=\boldsymbol{a}+\boldsymbol{k} \boldsymbol{n}_{\mathbf{j}}$.

\begin{tabular}{|c|c|c|c|c|c|c|c|c|}
\hline & $<n_{\mathrm{s}}>$ & ${ }^{2} \chi$ & $<n_{\mathrm{g}}>$ & ${ }^{2} \chi$ & $<n_{\mathrm{b}}>$ & ${ }^{2} \chi$ & $<n_{\mathrm{h}}>$ & ${ }^{2} \chi$ \\
\hline$n_{\mathrm{s}}$ & --- & --- & $0.23 \pm 14.02$ & 0.87 & $-0.07 \pm 15.6$ & 0.56 & $0.16 \pm 29.65$ & 0.95 \\
\hline$n_{\mathrm{g}}$ & $0.76 \pm 10.42$ & 0.96 & --- & & $0.25 \pm 19.6$ & 0.65 & $0.69 \pm 20.30$ & 0.95 \\
\hline$n_{\mathrm{b}}$ & $0.54 \pm 33.1-$ & 0.94 & $0.23 \pm 14.02$ & 0.84 & --- & --- & $0.58 \pm 27.09$ & 0.87 \\
\hline
\end{tabular}



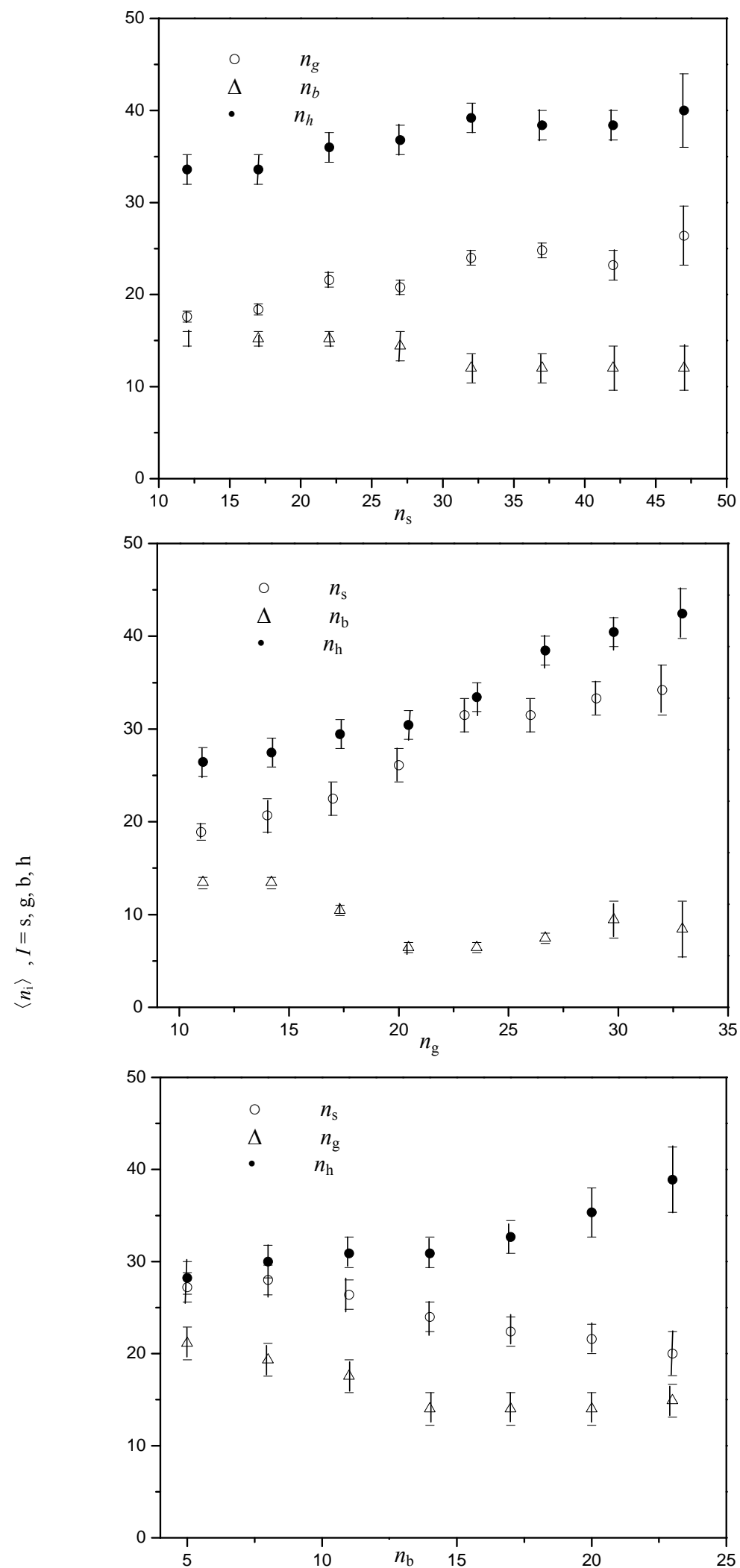

Figure 1. The correlations between the secondary particles multiplicities for complete destructions (events with $n_{\mathrm{h}}>28$ ) in $\mathrm{Si}^{28}$ interactions with emulsions. 


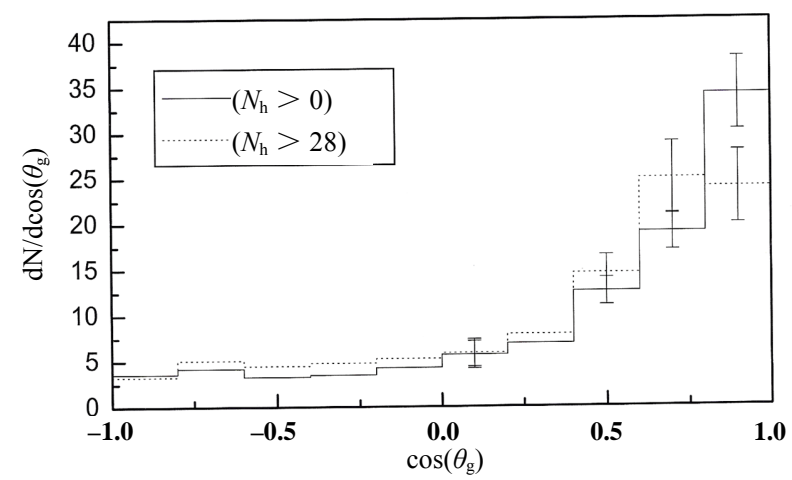

Figure 2. The angular distribution of grey particles emitted in central collision and all interactions of ${ }^{28} \mathrm{Si}$ ions with emulsion at $4.5 \mathrm{GeV} / \mathrm{c}$.

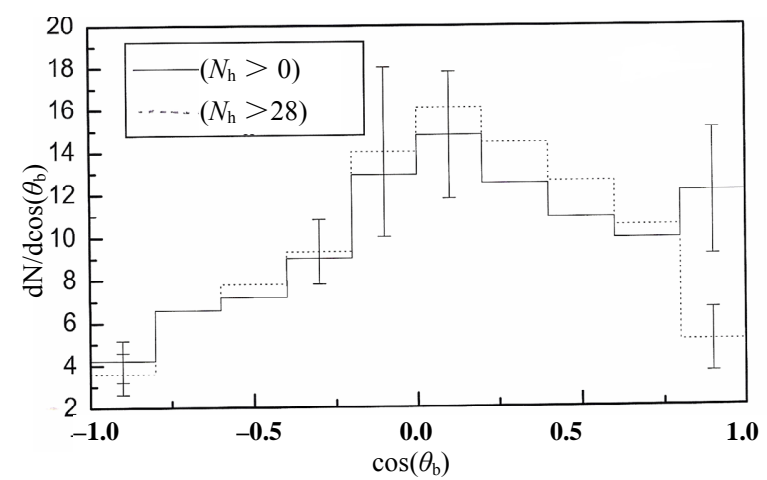

Figure 3. The angular distribution of black particles emitted in central collision and all interactions of ${ }^{28} \mathrm{Si}$ ions with emulsion at $4.5 \mathrm{GeV} / \mathrm{c}$.

angular distributions of black particle tracks, i.e. tracks with range $L<3 \mathrm{~mm}$ which is equivalent to proton kinetic energy $<27 \mathrm{MeV}$, emitted from central collisions Within statistical errors the distribution are consistent with each other. The target fragment angular distribution show on statistically significant peak which could be attributed to shock wave.

\section{Identification of Hadronic Clusters}

In the selected interaction, the shower particles mostly of kinetic energy $T>60 \mathrm{MeV}$ and a few protons of $T>400$ $\mathrm{MeV}$, were identified and their polar angles $\theta$ were measure. For each shower particle the pseudo rapidity $\eta$ was determined $[\eta=-$ In $\tan \theta / 2]$. The $\eta$ distribution was found to be nearly flat in the range of $\eta=-1$ to +1 . This enabled us to use quantitative method of pseudo rapidity intervals to search for clustering [16]. In each of the selected event we define $\Delta \eta_{i j}^{k}=\left|\eta_{\mathrm{i}}-\eta_{\mathrm{j}}\right|$, where $I \neq j \& i, j=1, \cdots, n$ and $k=0,1, \cdots, n-2$ ( $n$ is the number of shower particles between $\eta=-1$ and +1 in the given interaction ). The quantity $\Delta \eta_{i j}^{k}$ means the pseudo rapidity interval between the $i$-th and $j$-th particles such that $\mathrm{k}$ particles are lying between them. The value of $\dot{\eta}$ was rescaled in each event, i.e., $\eta=\left(\eta_{\mathrm{i}}-\eta_{1}\right) /\left(\eta_{\max }-\eta_{1}\right)$, such that is takes a value between zero and unity, where $\eta_{1}$ and all interactions of ${ }^{28} \mathrm{Si}$ ions with emulsion and $\eta_{\max }$ are the minimum and maximum value of that $\eta$. Figure 4. presents the frequency distribution of $\Delta \eta$. From this figure, it is seen that the maximum of the experimental delta is a $\Delta \eta=0.55$ while the maximum of the independent particle emission is at $\Delta \eta=0.65$. The distribution in Figure 4. was drawn for the number of particle in an interval of $k=19$ and the multiplicity of considered tracks is normalized to $n=30$. The histogram in Figure 4. is the experimental data, while the curve is for the IPE which is described by a binomial probability distribution and its form is given by

$$
\begin{aligned}
& \frac{\mathrm{d} N_{k}^{n}}{\mathrm{~d} \Delta \eta}=n C_{n-1}^{k} \Delta \eta^{k}(1-\Delta \eta)^{n-k-1} \\
& C_{n-1}^{k}=(n-1) ! / K !(n-k-1) !
\end{aligned}
$$

Where, the IPE distribution has a maximum at $\Delta \eta^{\prime} \max =$ $k / n-1$. It is seen that the experimental $\Delta \eta$-distribution is shifted to the left with respect to the IPE one. This shows that a big cluster of hadronic nature may be formed within the shower particles during their creation process.

\section{Identification of Baryonic Cluster}

It is interesting to investigate whether the hadronic clusters were accompanied by a baryonic one which is formed from target nucleus nucleons during the motion of the hadronic cluster inside the target nucleus. For this purpose, we used the method described in [17]. The method is based on the same principles used in searhpring for hadronic cluster. Firstly, we made sure that azimuthal angular distribution of grey particle ( $\psi$ - distribution) is isotropic. Then, in each, we define $\Delta \psi_{i j}^{k}=\left|\psi_{\mathrm{i}}-\psi_{\mathrm{j}}\right|$, where $I \neq j \& i, j$ $=1,2, \ldots, n_{\mathrm{g}}\left(n_{\mathrm{g}}\right.$ is the number of grey particles in the event) and $k=0,1, \ldots, n_{\mathrm{g}}-2$. The quantity $\Delta \psi_{i j}^{k}$, means the azimuthal angular interval between the $\underline{i}$-th and $j$-th grey particle such that $k$ of grey particle are lying between them. Figure 5. show the $\Delta \psi_{\mathrm{g}}$ distribution for ${ }^{28} \mathrm{Si}+\mathrm{AgBr}$ reaction the first maximum is at $\Delta \psi_{\mathrm{g}}=0.15$ and the corresponding IPE is at $\Delta \psi_{\mathrm{g}}=0.35$. This figure is drawn for a number of grey in an interval $k=10$ for ${ }^{28} \mathrm{Si}+\mathrm{AgBr}$ interactions while the multiplicity of considered tracks is normalized to $n=30$. The histogram is the experimental data while curve is due to IPE. As shown in Figure 5. experimental $\Delta \psi_{\mathrm{g}}$-distribution is shifted to the left with respect to IPE one. This can be interpreted as formation of a baryonic cluster within the grey particles of the target fragments.

It is interesting to investigate whether the effect of clustering may appear within the black-particles. For this purpose we repeated the same analysis for black tracts. 


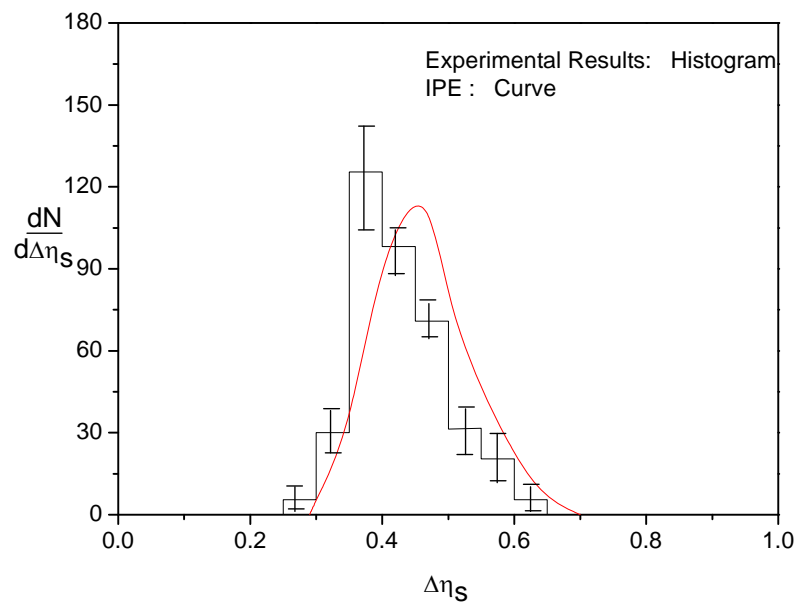

Figure 4. The frequency distribution of pseudo rapidity interval $(\Delta \eta$-distribution ).

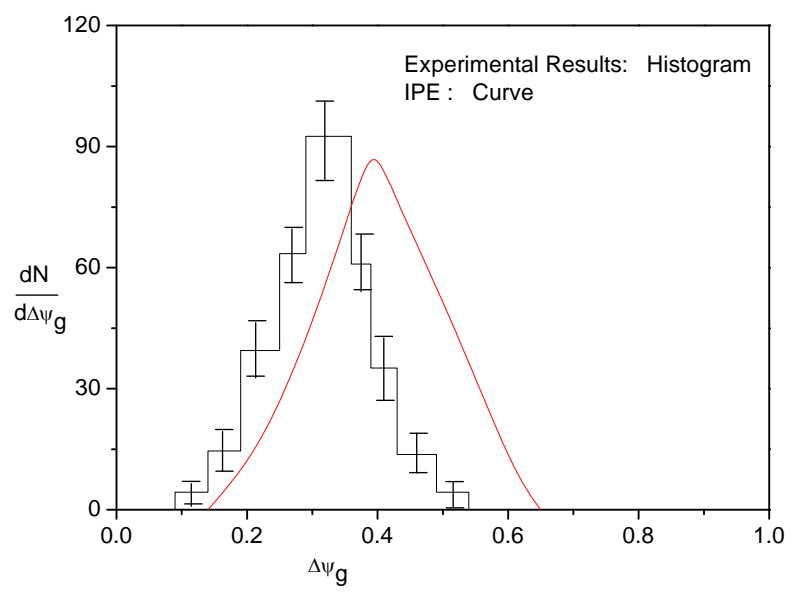

Figure 5. The distribution of azimuthal angular interval for grey particles in ${ }^{28} \mathrm{Si}+\mathrm{AgBr}$.

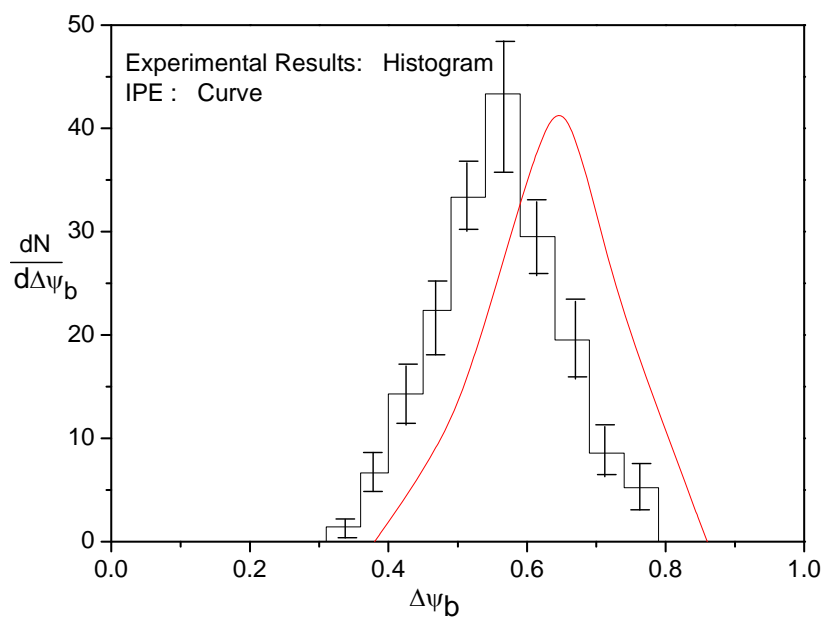

Figure 6. The distribution of azimuthal angular interval for black particles in ${ }^{28} \mathrm{Si}+\mathrm{AgBr}$.
Figure 6. shows the $\Delta \Psi_{\mathrm{b}}$ - distribution for ${ }^{28} \mathrm{Si}+\mathrm{AgBr}$ reactions at $\Delta \psi_{\mathrm{g}}=0.15$ and the corresponding IPE maximum is at $\Delta \psi_{\mathrm{b}}=25$ this figure is drawn for a number of particle $k=4$ while the multiplicity of the considered tracks is normalized to $n=16$. The histograms represent the experimental data while the curve is due to IPE. It is seen that, the experimental $\Delta \psi_{\mathrm{b}}$-distribution is shifted to left with respect to the IPE one. This may be interpreted as formation of a baryonic cluster.

\section{Conclusions}

From the investigation of particles emitted in the central ${ }^{28} \mathrm{Si}$-emulsion collisions, we can make the following conclusions.

1) From the above results, one can that $\left\langle n_{\mathrm{b}}\right\rangle$ has a negative correlation with $n_{\mathrm{s}}$ and $n_{\mathrm{g}}$ and the correlation of $<n_{\mathrm{s}}>$ and $<n_{\mathrm{g}}>$ with the $n_{\mathrm{b}}$ is negative .Thus the correlation in complete destruction are often different from these total sample.

2) The angular distributions of target fragments are independent on the projectile mass, the impact parameter and one the target nucleus.

3) The target fragments angular distribution show no statistically significant peak which could be attributed to shock wave.

4) The ns-distribution, from central ${ }^{28} \mathrm{Si}+\mathrm{AgBr}$ collisions, is nearly a poission one which is narrower than the corresponding distributions from $\mathrm{pp}$ and $\mathrm{p}$-nucleus interactions.

5) The emission of $s, g, b$, particles dose not agree with independent particle emission (IPE).

Using the $\Delta$ method an interacorrelation is observed within s, g, b, particle with maybe interpreted as formation of clusters of these particles.

\section{References}

[1] D. Beavis, S. Y. Fung, W. Gorn, D. Keano, Y. M. Liu, R. T. Poe. Van. Dalen and M. Vient, "Spatial Self-Organization of Surface Structure during an Oscillating Catalytic Reaction," Physical Review Letters, Vol. 54, No. 15, 1985, pp. 1652-1655. doi:10.1103/PhysRevLett.54.1652

[2] P. Beckkmann, H. A. Gustafsson, H. H. Gutbrod, K. H. Kalmpert, B. Kolb, H. Loethren, A. M. Poskanzer, H. G. Ritter, H. R. Schmidt and T. Siemiacczck, "Collective Azimuthal Align ment in Reletivistic Heavy ion Reactions,"Physical Letters A., Vol. 2, 1987, pp. 163-168.

[3] L. P. Csernai, P. Freier, J. Mevissen, H. Nguten and L. Waters, "Identification of Collective Flow by Transverse-Momentum Analysis of Emulsion Data for $\mathrm{Au}+$ $\mathrm{AgBr}$ and $\mathrm{Xe}+\mathrm{AgBr}, "$ Physical Review C, Vol. 34, 1986, pp. 1270-1273. doi:10.1103/PhysRevC.34.1270

[4] D. Beavis, et.al., "Expanding Pion Emitting Source in Ar 
on Pb Collisions," Physical Review, Vol. 34, No. 2, 1986, pp. 757-760.

[5] S. Z. Belenkij and L. D. Landau, "Relating Multihadron Production in Hadronic and Nuclear Collisions,"SSP Fiz -Nauk, Vol. 56, 1955, p. 309.

[6] T.-C Meny, "Institute Für Theroretische Physic Der Freien Universtat,” Berlin Report Fub, HEP., 1978.

[7] Y. Alek, et.al, "Theory of Two-Dimensional Melting," Physical Review Letters, Vol. 41, No. 2, 1978, pp.122124. doi:10.1103/PhysRevLett.41.849

[8] J. P. Vary, "Multiple-Collision Model for Pion Production in Relativistic Nucleus Collisions," Physical Review Letters, Vol. 40, No. 5, 1978, pp. 295-298. doi:10.1103/PhysRevLett.40.295

[9] V. S. Barashenkov, V. A. Belyakov and Shu-Fen Van, "Cross Sections for Elementary Particle Interactions,"Communication of JINR PI-331, Dubna, 1959.

[10] A. M. Baldin, et. al., "Cumulative Production of Particles in High Energy Collisions,"International Seminar On High Energy physical D1-2-84-559, Dubna, 1984, p. 531.

[11] B. K. Kalinkinet, et. al., "Recent Experiments at Brook-
Haven: Level Structure of $N=86$ Isotones ${ }^{156} \mathrm{Yb}$ and ${ }^{150}$ Gd," Physica Scripta, Vol. 24, No. 1B, 1981, p. 298. doi:10.1088/0031-8949/24/1B/027

[12] N. N. Abd-Allah, "Complete destruction of $\mathrm{Ag}(\mathrm{Br})$ Emulsion Nuclei Induced by $4.5 \mathrm{~A} \mathrm{GeV} / \mathrm{c} \mathrm{Si}^{28}$ ions," Physica Scripat, Vol. 47, No. 4, 1993, pp. 501-504.

[13] K. D. Tolestov, "Central heavy-ion collisions with emulsion at $4.2 \mathrm{GeV} / \mathrm{c}$ per incident nucleon,"JJNB Dubna EI-80-522, 1980.

[14] B. Otterund, "High Energy Hadron Reactions on Nuclei and High Energy Heavy Ion Reactions," Cairo University, Egypt, 1979.

[15] K. D. Tolestov, "Complete destruction of heavy nuclei by hadrons and nuclei,"JINR Dubna, PI-8662, 1975.

[16] E. Nagly, et al., "Fragmentation of ${ }^{28} \mathrm{Si}$ nuclei in nuclear emulsion," JINR Preprint EL-87-546, Dubna, 1987.

[17] B. P. Bannik, et al., "Observation of a Collective Flow in $^{22} \mathrm{Ne}$ Collisions with $\mathrm{Ag}(\mathrm{Br})$ Emulsion Nuclei at $4.1 \mathrm{~A}$ GeV/c," Zeitschrift Fur Physical A Hadrons and Nuclel, Vol. 329, No. 3, 1988, pp. 341-347. doi:10.1007/BF01290239 